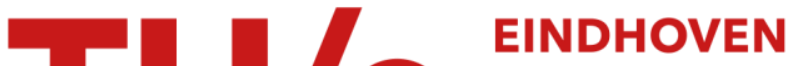

\section{Stabilization of protein-protein interactions by small molecules}

Citation for published version (APA):

Giordanetto, F., Schäfer, A., \& Ottmann, C. (2014). Stabilization of protein-protein interactions by small molecules. Drug Discovery Today: Technologies, 19(11), 1812-1821.

https://doi.org/10.1016/j.drudis.2014.08.005

DOI:

10.1016/j.drudis.2014.08.005

Document status and date:

Published: 01/01/2014

\section{Document Version:}

Publisher's PDF, also known as Version of Record (includes final page, issue and volume numbers)

\section{Please check the document version of this publication:}

- A submitted manuscript is the version of the article upon submission and before peer-review. There can be important differences between the submitted version and the official published version of record. People interested in the research are advised to contact the author for the final version of the publication, or visit the $\mathrm{DOI}$ to the publisher's website.

- The final author version and the galley proof are versions of the publication after peer review.

- The final published version features the final layout of the paper including the volume, issue and page numbers.

Link to publication

\section{General rights}

Copyright and moral rights for the publications made accessible in the public portal are retained by the authors and/or other copyright owners and it is a condition of accessing publications that users recognise and abide by the legal requirements associated with these rights.

- Users may download and print one copy of any publication from the public portal for the purpose of private study or research.

- You may not further distribute the material or use it for any profit-making activity or commercial gain

- You may freely distribute the URL identifying the publication in the public portal.

If the publication is distributed under the terms of Article 25fa of the Dutch Copyright Act, indicated by the "Taverne" license above, please follow below link for the End User Agreement:

www.tue.nl/taverne

Take down policy

If you believe that this document breaches copyright please contact us at:

openaccess@tue.nl

providing details and we will investigate your claim. 


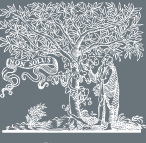

ELSEVIER

\section{Stabilization of protein-protein interactions by small molecules}

\section{Fabrizio Giordanetto ${ }^{1}$, Anja Schäfer ${ }^{2}$ and Christian Ottmann ${ }^{2}$}

\footnotetext{
${ }^{1}$ Medicinal Chemistry, Taros Chemicals, Emil-Figge-Str. 76a, 44227 Dortmund, Germany

${ }^{2}$ Laboratory of Chemical Biology and Institute of Complex Molecular Systems, Department of Biomedical Engineering, Technische Universiteit Eindhoven, Den Dolech 2, 5612 AZ Eindhoven, The Netherlands
}

Protein-protein interactions (PPIs) are implicated in every disease and mastering the ability to influence PPIs with small molecules would considerably enlarge the druggable genome. Whereas inhibition of PPIs has repeatedly been shown to work successfully, targeted stabilization of PPIs is underrepresented in the literature. This is all the more surprising because natural products like FK506, rapamycin, brefeldin, forskolin and fusicoccin confer their physiological activity by stabilizing specific PPIs. However, recently a number of very interesting synthetic molecules have been reported from drug discovery projects that indeed achieve their desired activities by stabilizing either homo- or hetero-oligomeric complexes of their target proteins.

\section{Introduction}

Small molecule modulation of protein-protein interactions (PPIs) has been recognized as a promising approach in drug discovery [13]. However, in the vast majority of cases, PPI modulation is exclusively recognized as inhibition, understandably motivated by a number of wonderful success stories that have been published in recent years. Of these, two of the most prominent examples are the nutlins developed by Roche $[4,5]$, which inhibit the negative regulation of the tumor suppressor p53 by the ubiquitin ligase mouse double minute 2 homolog (MDM2), and navitoclax from Abbott (ABT263), which disrupts the interaction of the antiapoptotic protein Bcl-2 and apoptosis-executing proteins like Bad, Bid and Bak [6-8]. Further prominent examples are molecules that inhibit the interaction of human leukemia-derived growth factor (LDGF) with HIV integrase [9], disrupt the binding of KRas and phosphodiesterase (PDE) $\delta$ [10], transform an active tumor necrosis factor (TNF) $\alpha$ trimer into an inactive dimer [11] or inhibit the binding of PPI modules like the clathrin terminal domain (CTD), or bromodomains with their partners $[12,13]$.

A number of natural products have been shown to mediate their physiological activity by stabilizing PPIs, for a review see [14]. These molecules are ample proof of the concept of small molecule PPI interface binding which results in functional stabilization of

Corresponding author:. Ottmann, C. (c.ottmann@tue.nl) regulatory protein complexes leading to significant physiological effects. In addition to these examples from nature itself, there are meanwhile a number of small molecules known that have been found to modulate the function of their protein target by stabilizing their homo or hetero complexes. Interestingly, many of them have been identified during screening of PPI inhibitors, for example RO2443 and phenothiazines that inhibit the function of MDMX and the protein S100A4, respectively. A few molecules have also been found in approaches directly dedicated to identifying PPI stabilizers, for example pyrrolidone1. In this review we summarize the current state of PPI stabilization by small molecules, including natural products and synthetic molecules.

\section{Cyclosporin A}

Cyclosporin A (CsA) is a cyclic undecapeptide produced by the ascomycete Tolypocladium inflatum, initially isolated in 1970 from a Norwegian soil sample and shown to display immunosuppressive properties [15]. Its chemical structure inclusive of $N$-methylated peptide bonds, the atypical amino acids aminobutyric acid and $(4 R)$ 4-[(E)-2-butenyl]-4,N-dimethyl-L-threonine (MeBmt) and D-alanine is biosynthesized by a nonribosomal process [16] (Table 1). The conformational, physicochemical and pharmacokinetic properties of CsA, most notably its oral absorption profile [17], continue to inspire chemists [18-20], and its effective immunosuppressant action supported FDA registration in 1983 [21]. The molecular target 
TABLE 1

Natural products that stabilize protein-protein interactions (PPIs)
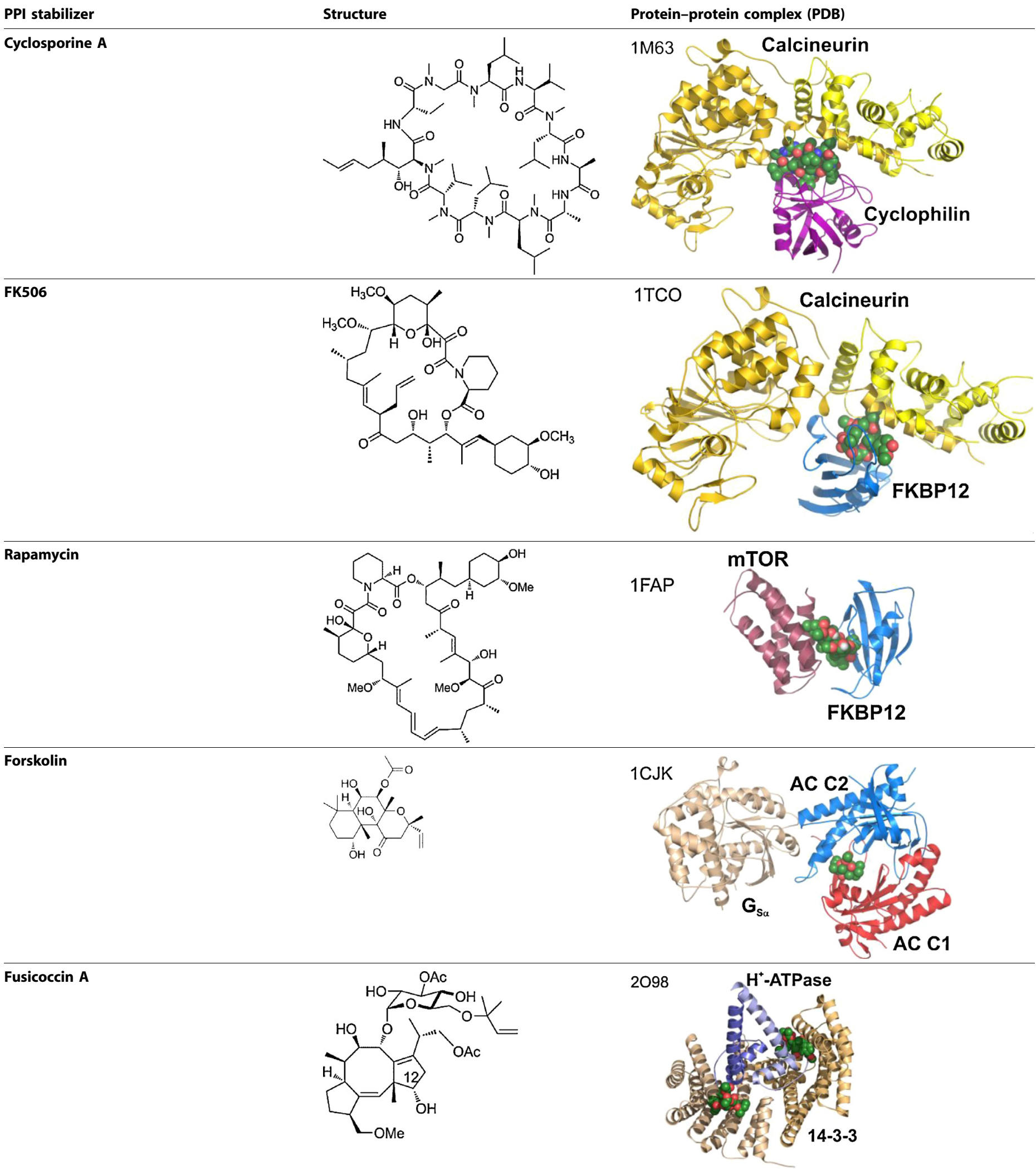

mTOR 
TABLE 1 (Continued)

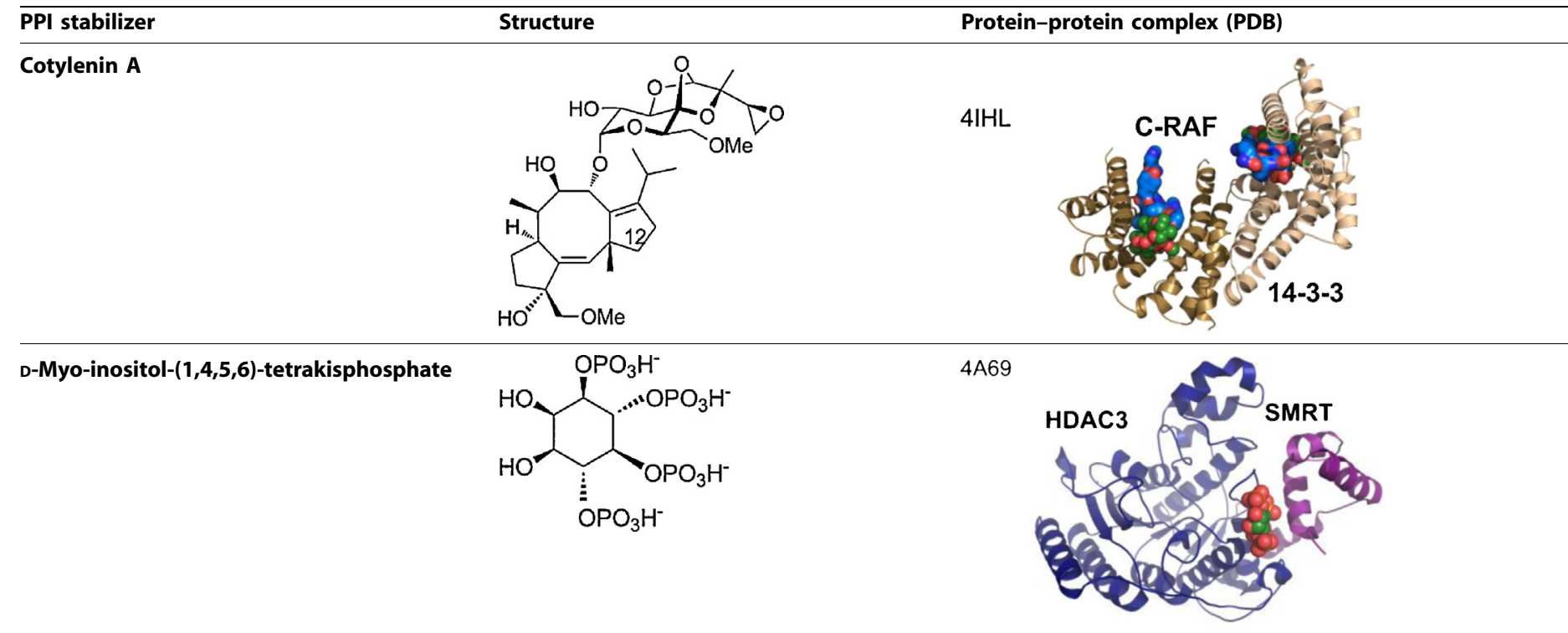

of CsA was identified one year later to be cyclophilin A (CypA) [22]. Direct binding to this peptidyl-prolyl cis-trans isomerase leads to inhibition of its enzymatic activity explaining the usefulness of CsA derivatives for antiviral therapy [23]. However, the functionally relevant target for the immunosuppressive activity of CsA is not the individual CypA protein but rather its complex with the phosphatase calcineurin (Cn) [24]. Elucidation of the corresponding crystal structure of the ternary complex Cn-CypA-CsA showed how CsA inhibits the enzyme activity of $\mathrm{Cn}$ by 'gluing' CypA to the phosphatase and blocking the access to the active site [25]. Two further molecules, entirely unrelated to CsA, have also been found to display immunosuppressive activity: the macrolides FK506 and rapamycin.

\section{FK506 (tacrolimus)}

FK506 was isolated by scientists at Fujisawa Pharmaceuticals in 1984 as the active immunosuppressant component of a fermented broth from Streptomyces tsukubaensis [26]. FK506 is a 23-membered lactone belonging to the ascomycin macrolide class; it contains 14 asymmetric centers, nine sites of oxygenation and one amino acid, making targeted medicinal chemistry efforts a formidable challenge [27]. FK506 (tacrolimus) was approved as an oral immunosuppressant drug to be used after allogeneic transplant in 1994. The direct target for FK506 is the peptidyl-prolyl isomerase FK506 binding protein (FKBP) [28]. Very similar to CsA, also the effective physiological target of FK506 is not FKBP alone but a complex of the FKBP-bound FK506 and the phosphatase calcineurin [24]. Similar to the situation with CsA-CypA, the FK506-FKBP complex inhibits calcineurin by obstructing the active site of the phosphatase as shown in the crystal structure of the ternary complex published by scientists from Vertex [29].

\section{Rapamycin}

The third molecule that was found to have profound immunosuppressive activity and therefore helped to revolutionize transplantation medicine was rapamycin. This natural product was initially described as an antifungal metabolite from Streptomyces hygroscopicus, isolated from a soil sample of the island of Rapa Nui [30]. It was found shortly afterward to have inhibitory activity toward the mammalian immune system [31]. Rapamycin is a 31-membered lactone with 15 chiral centers, an all-trans triene and a hemiketal masked tricarbonyl system (Table 1). Exactly like FK506, rapamycin binds to FKBP but instead of attaching to and inhibiting calcineurin the rapamycin-FKBP complex inhibits the kinase mammalian target of rapamycin (mTOR) [32] with mechanistic details revealed by the crystal structure of the ternary complex [33].

The molecular interactions established by rapamycin with FKBP12 and mTOR are in line with previously reported SAR studies including modifications at the C7 [34], C16 and C20 positions [35]. Consistent with the crystal structure of the FKBP12-mTORrapamycin complex, modification at the C-43 position of rapamycin is neutral to the biological activity and serves mainly to modulate physicochemical and pharmacokinetic properties of the drug, as highlighted by the marketed rapalogs [36,37]. In all three cases - CsA, FK506 and rapamycin - the resulting inhibitory complexes are established only in the presence of the small molecule, illustrating the impressing PPI-stabilizing properties of these compounds.

\section{Forskolin}

Forskolin (Fsk) was isolated in 1977 from Coleus forskohlii and researchers from Hoechst described it as an inotropic and bloodpressure-lowering compound [38]. Fsk achieves this activity by increasing the level of the second messenger molecule cAMP through activation of adenylyl cyclase [39]. Here, Fsk stabilizes the interaction of the C1 and C2 subunit of adenylyl cyclase, thereby enhancing the activity of this enzyme [40]. In the crystal structures of either the C2 homodimer [41] or the C1-C2 heterodimer complexes with the stimulatory $\alpha$ subunit of a trimeric $G$ protein $(\mathrm{Gs} \alpha)$ [42], Fsk binds to a composite pocket in the rim of the dimer interface. This binding site is strongly hydrophobic and buries $90 \%$ of the solvent-accessible surface of Fsk. Stabilization of the PPI is achieved by filling this gap in the interface and eliminating the unfavorable hydration of the interior surface of the pocket. Chemically, Fsk (17- $\beta$-acetoxy-8,13-epoxy- $1 \alpha, 6-\beta, 9 \alpha$-trihydroxy-labd-14-en-11-one) is a labdane diterpene, containing 
an atypical pyrane ring (Table 1). Because of its cardiovascular properties, Fsk has been the subject of several chemical optimization efforts, with special emphasis on improving its aqueous solubility [38,43-46]. Interestingly, none of the Fsk derivatives synthesized to date proved superior to the natural product with respect to in vivo antihypertensive and inotropic effects.

\section{The 14-3-3 PPI stabilizers fusicoccin A and cotylenin A}

The two natural products fusicoccin A and cotylenin A were first described as agents acting on plants $[47,48]$, but were later found to show activities toward human cells [49-51]. For example, fusicoccin A shows antitumor activity [52], promotes platelet aggregation [53] and stabilizes the inhibitory binding of 14-3-3 to $\mathrm{ER} \alpha$ [54]. Cotylenin A has been shown to induce differentiation in myeloid leukemia [55], display activity against breast cancer cells [56] and stabilize the inhibitory binding of 14-3-3 proteins to c-Raf $[57,58]$. We have furthermore shown that a semisynthetic derivative of fusicoccin - FC-THF - enhances transport of the potassium channel TASK3 to the plasma membrane by stabilizing 14-3-3 binding to the C terminus of TASK3 [59]. Taken together, the fusicoccanes are a valuable class of natural products for the targeted stabilization of the widespread 14-3-3 interactome with modifications - either by semi-synthesis $[59,60]$ or by recombinant fermentation $[61,62]$ potentially resulting in specific compounds. Given the fact that 14-3-3 proteins interact with hundreds of different proteins in eukaryotic cells [63], this issue of specificity is a pivotal one and stands in the focus of current efforts to develop new molecules for the modulation of the functions of this family of adapter proteins.

\section{Inositol tetraphosphate}

In humans, D-myo-inositol 1,4,5,6-tetrakisphosphate [Ins(1,4,5, 6) $\mathrm{P}_{4}$; Table 1] is produced by inositol phosphate multikinase (IMPK) that phosphorylates $\operatorname{Ins}(1,4,5) \mathrm{P}_{3}$ to form $\operatorname{Ins}(1,3,4,5,6) \mathrm{P}_{4}$ [64]. The yeast homolog of IMPK - the kinase Arg82 - has been shown to be involved in transcriptional regulation suggesting a functional role of Ins $(1,3,4,5,6) \mathrm{P}_{4}$ in regulation of gene expression. Furthermore, Ins $(1,3,4,5,6) \mathrm{P}_{4}$ is elevated during Salmonella enterica infection [65].

In 2012, Watson et al. [66] published the crystal structure of histone deacetylase 3 (HDAC3) in complex with the transcriptional co-repressor silencing mediator of retinoid and thyroid hormone receptors (SMRT). To the researchers' surprise they observed clear electron density of $\operatorname{Ins}(1,3,4,5,6) \mathrm{P}_{4}$ in the rim of the interface of the two proteins, obviously being picked up by the protein complex from its host expression system, HEK293 cells (human embryonic kidney cells). In contrast to the predominantly hydrophobic binding sites of FK506, rapamycin and forskolin, the pocket of Ins $(1,3,4,5,6) \mathrm{P}_{4}$ displays a strong basic character. Importantly, the HDAC3-SMRT complex is not stable in the absence of the molecule, suggesting an important functional role of Ins $(1,3,4,5,6) \mathrm{P}_{4}$ in transcriptional repression by HDAC3.

\section{Dexrazoxane}

Bisdioxopiperazines are small chemical compounds that inhibit the activity of DNA topoisomerase type II [67]. Among these remarkably simple molecules, (S)-4,4'-(1-methyl-1,2-ethanediyl)bis-2,6-piperazinedione (dexrazoxane, ICRF-187; Table 2) has been approved for use in cancer patients to prevent anthracycline-mediated cardiotoxicity [68]. Intriguingly, dexrazoxane inhibits DNA topoisomerase II by stabilizing the closed conformation of the homodimeric enzyme [69]. Elucidation of the crystal structure for the topoisomerase II dimer complexed with dexrazoxane indicated how dexrazoxane bound to a composite, symmetric pocket in the interface of the topoisomerase II homodimer and contacts both monomers simultaneously at the exact corresponding sites in the antiparallel dimer [70].

\section{Tafamidis}

Aggregation of transthyretin (TTR) is known to be responsible for TTR amyloidoses leading to peripheral neuropathy and cardiomyopathy. The stabilization of proteins involved in misfolding processes associated to disease states was proposed to be an effective therapeutic strategy [71]. In this context, TTR amyloidosis has received widespread attention and several chemical classes of TTR kinetic stabilizers have been reported to date [72]. Among these, a benzoxazole series [73] was further optimized to 2-(3,5-dichlorophenyl)-benzoxazole-6-carboxylic acid (tafamidis; Table 1) [74], a potent TTR stabilizer that has been approved in Europe and Japan for the treatment of transthyretin-related hereditary amyloidosis. Tafamidis binds selectively to TTR variants and stabilizes the TTR dimer interface [74]. As can be seen from the crystal structure, tafamidis bound to TTR in a symmetric pocket established at the interface of the TTR. A hydrophobic subpocket is formed by the side-chains of Ala108, Leu110 and Thr112 and accommodates the 3,5-dichlorobenzene substituent, and the carboxyl group is engaged via water-mediated H-bonds with Lys15 and Glu54. Of clinical importance, tafamidis also stabilizes the most prevalently mutated TTR forms V30M and V122I.

\section{Phenothiazines}

Garrett et al. reported in 2008 the identification of several compounds that blocked the activity of S100A4 [75]. These inhibitors belong to the renowned phenothiazine chemical class, a family of compounds endowed with antipsychotic, antiallergic and anesthetic properties [76,77], resulting in breakthrough drugs such as chlorpromazine [78]. One of these compounds, trifluoperazine \{TFP, 10-[3-(4-methylpiperazin-1-yl)propyl]-2-(trifluoromethyl)$10 \mathrm{H}$-phenothiazine; Table 1$\}$, was later shown in a crystallographic and biochemical study to inhibit S100A4 function by stabilizing an inactive pentamer [79]. In the crystallized pentamer two TFP molecules are sitting adjacent to each other in the interface contributed from each monomer, adding up to four copies of TFP that make extensive mutual contacts and thus stabilize the oligomeric assembly.

\section{O-Methoxy-nucleozin}

Influenza nucleoprotein (NP) is an essential viral factor, necessary for replication and virion assembly [80]. Hence, inhibitors of NP function could be of interest as therapeutic agents. The groups of Yuen and Wong reported the identification of nucleozin as an NP inhibitor with antiviral activity [81,82]. In 2011, researchers at Bristol Myers Squibb identified related compounds via highthroughput screening [83]. Here, expansion of the original hit nucleozin compound via substructure searches identified a series of compounds with improved properties. Among these, orthosubstitution of the 3-phenyl ring of the nucleozin isoxazole ring 
TABLE 2

\section{Synthetic compounds that stabilize protein-protein interactions (PPIs)}

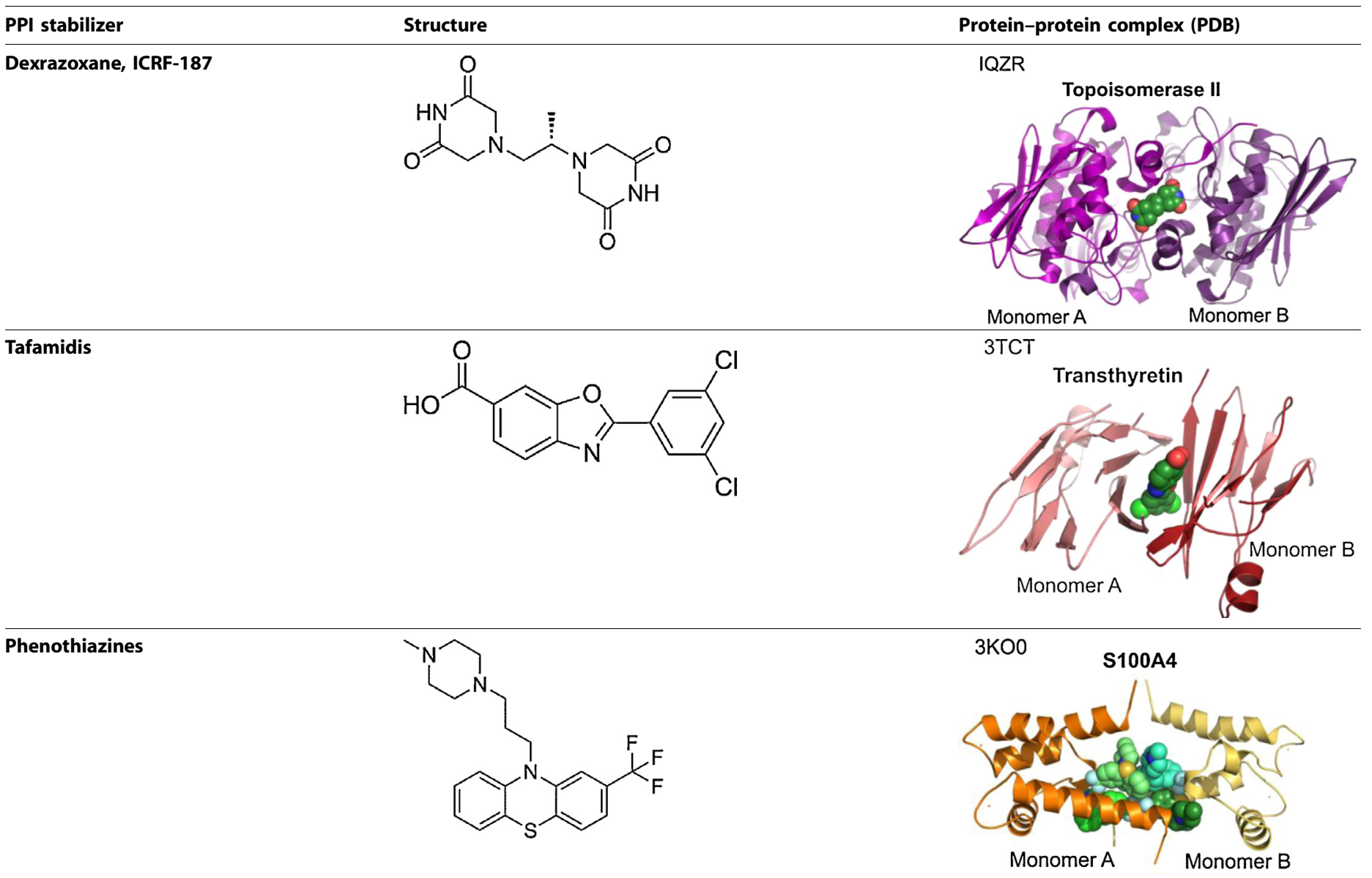<smiles>COc1ccccc1-c1noc(C)c1C(=O)N1CCN(c2ccc([N+](=O)[O-])cc2Cl)CC1</smiles>

3RO5

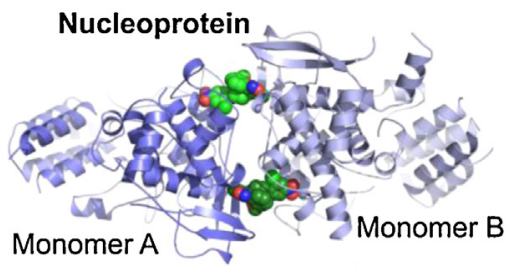

RO2443<smiles></smiles>

$3 \cup 15$

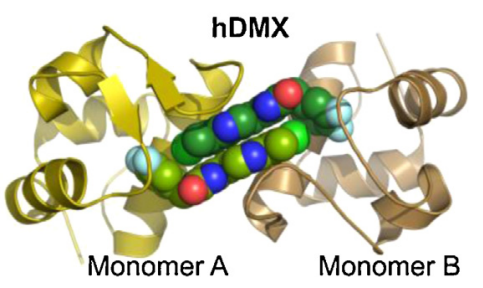

1EBIO<smiles>CCn1c(=O)[nH]c2ccccc21</smiles>

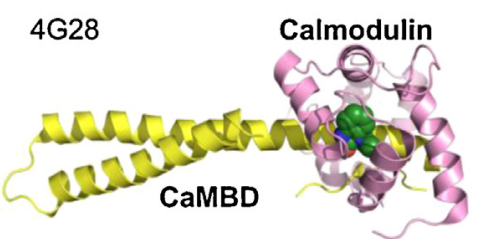


TABLE 2 (Continued)

PPI stabilizer

Epibestatin
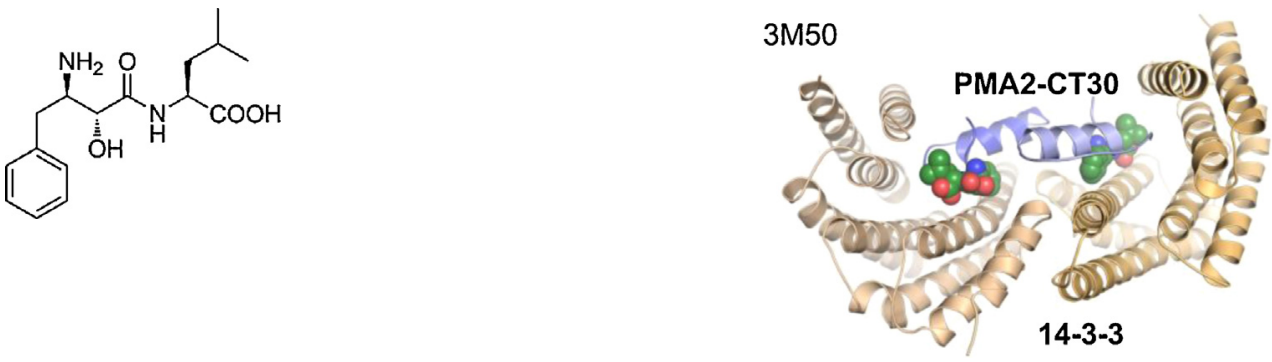

Pyrrolidone 1
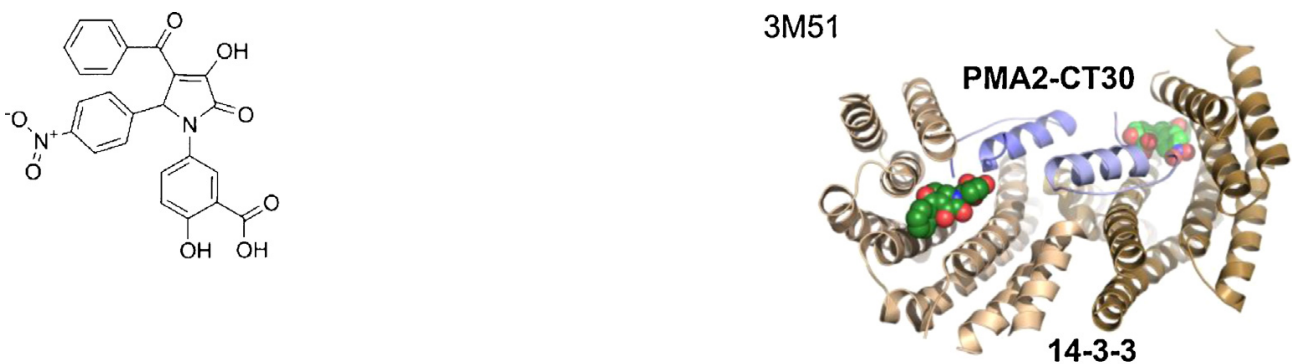

was preferred and (4-(2-chloro-4-nitrophenyl)piperazin-1-yl)(3-(2methoxyphenyl)-5-methylisoxazol-4-yl)methanone (O-methoxynucleozin; Table 1) was the most potent inhibitor [83]. Interestingly, scaffold hopping from isoxazole to 1-methyl-1H-1,2,3-triazole was also tolerated. O-Methoxy-nucleozin was shown by protein crystallography to bind in the interface at the NP oligomer, bridge the dimer interface and thus stabilize the protein complex.

\section{R02443}

p53, possibly the most important tumor suppressor in the human body, is negatively regulated by MDM2 and MDMX [84]. Whereas inhibition of binding of MDM2 to p53 has successfully been achieved by several small molecules [85], no such specific molecules have been reported for MDMX. However, to take full advantage of the strategy to stabilize p53 function, MDM2 and MDMX need to be targeted [86]. Such dual-activity inhibitors were identified by high-throughput screening by scientists at Roche and belonged to the class of indolyl-hydantoins [87]. Surprisingly, biochemical experiments and the co-crystal structure of MDMX or MDM2 in complex with one of these compounds, (5Z)-5-[(6chloro-7-methyl-1H-indol-3-yl)methylidene]-3-(3,4-difluorobenzyl)imidazolidine-2,4-dione (RO2443; Table 1), revealed that RO2443 bound to the interface of the homodimers and stabilized their interaction. This enhancement of either the MDMX or MDM2 homodimer together with a possible stabilization of the MDMX-MDM2 heterodimer includes parts of the interface with p53 and consequently excludes simultaneous binding to the tumor suppressor protein.

\section{$1 E B I O$}

1EBIO, a surprisingly simple benzoimidazolone (Table 1), was found to stabilize calmodulin-potassium-channel interaction. Calmodulin (CaM) is a protein tethered to small-conductance potassium channels (SK) as a $\mathrm{Ca}^{2+}$ sensor. Binding of $\mathrm{Ca}^{2+}$ to 
CaM leads to opening of the SK channel [88]. This opening in turn leads to reduced activation potential and vasodilatation. Abnormalities in SK channel function have been linked to schizophrenia and hypertension $[89,90]$. After finding that phenylurea, an additive in the crystallization solution, sat in the interface between CaM and SK, Zhang et al. co-crystallized the small molecule 1EBIO with CaM-SK [91]. It was shown that the known SK channel modulator 1EBIO occupies a pocket between the two proteins acting as a molecular glue. 1EBIO itself is a weak stabilizer, the structurally closely related NS309 (6,7-dichloro-3-(hydroxyimino)indolin-2-one) is 1000-times more-potent in augmenting current in whole-cell HEK293 patch clamp assays and its action could be antagonized by the SK blocker apamin [92,93].

\section{Stabilizers of nuclear-receptor-corepressor interactions}

Most nuclear receptors (NR) are ligand-activated transcription factors. In general, they can be activated by agonists or blocked by 'passive' antagonists [94]. In addition, antagonists can also be inverse agonists actively recruiting co-repressors. During the past decade, several interesting crystal structures of NR in complex with antagonists were solved. Two important co-repressor proteins that will be discussed below are nuclear receptor co-repressor (N-CoR) and silencing mediator of retinoid and thyroid hormone receptors (SMRT).

Asoprisnil (J867) [95] is a steroidal selective progesterone receptor (PR) modulator (SPRM) in clinical development against endometriosis. It displays mostly antagonist behavior but also weak agonist effects in fluorescence polarization and mammalian twohybrid assays. Two crystal structures of asoprisnil in the ligandbinding pocket of the PR with SMRT or N-CoR, respectively, were solved showing that the molecule is, with its hydrophilic $\mathrm{NOH}$ group, in direct contact with the lipophilic side-chain of Leu2263 in the SMRT peptide (3.16 ̊) [96]. Hence, changing the $\mathrm{NOH}$ group could lead to hydrophobic interaction between co-repressor and small molecule. Also, asoprisnil strongly recruits the co-repressor N-CoR, but only weakly recruits SRC-1.

An additional example of NR ligands facilitating co-repressor binding is the propynylbenzene bearing pan-retinoic acid receptor (RAR) inverse agonist BMS493 (Table 1). The compound strongly increases interaction of all RARs $(\alpha, \beta, \gamma)$ with SMRT and N-CoR. This was first shown by electrospray ionization (ESI)-MS for RAR homodimers and RAR-retinoic-X-receptor (RXR) heterodimers [97]. The dissociation constant $\left(K_{\mathrm{d}}\right)$ in a fluorescence polarization assay shifted from $3.8 \mu \mathrm{M}$ (agonist Am80) to $0.18 \mu \mathrm{M}$ for a rhodamine-labeled SMRT peptide, whereas the $K_{\mathrm{d}}$ for a fluoresceinlabeled SRC-1 peptide increased from $0.47 \mu \mathrm{M}$ (Am80) to 12.7 (BMS493). A year later, the same group was able to solve the crystal structure of BMS493, RAR $\alpha$ and a N-CoR 19mer peptide [98]. The compound is accommodated in the ligand-binding pocket making new contacts with Ile396 and Leu398, which changes the $\alpha$-helix 11 of RAR into a $\beta$-strand that can interact well with a $\beta$-strand in the N-CoR peptide, an occurrence that has not been observed in a NR crystal before. In addition to contacts with RAR, BMS493 also makes hydrophobic contacts with Leu2051, His2054, Ile2055 and, to a lesser extent, Ile2058 of the N-CoR peptide, hence it qualifies as a true interface binding stabilizer of PPI. BMS493 could be used as a medicinal chemistry starting point for the development of subtype selective ligands.

\section{Epibestatin and pyrrolidone1}

The phytotoxic activity of fusicoccin A could in principle qualify this compound as a promising developmental candidate for a new total herbicide. However, the complex chemical nature and high costs of production by fermentation hampers its use in plant protection. Because the binding pocket of fusicoccin in the 143-3-PMA2 complex has the potential to accommodate less chemically demanding molecules we screened a mid-sized (37 000) small molecule library for stabilizers of the 14-3-3-PMA2 interaction and identified the dipeptide epibestatin and a pyrrolinone (pyrrolidone1) as significantly more amenable functional substitutes for fusicoccin [99]. The corresponding crystal structures showed that the two molecules bound to different sites in the complex interface; whereas epibestatin binds to a narrow pocket between 14-3-3 and PMA2 and is literally 'sandwiched' in a narrow cleft between the two proteins, pyrrolidone1 binds to a relatively open pocket. This binding behavior is reflected in the dynamics of the stabilizing activity of these two compounds with pyrrolidone 1 mainly enhancing the association rate of the 14-3-3-PMA2 complex and epibestatin mainly decreasing the dissociation rate [99].

\section{Concluding remarks}

PPIs are normally regarded as less tractable than other more established protein target families [e.g. kinases, G-protein-coupled receptors (GPCRs), ion channels]. Their inhibition using small molecules is considered technically challenging owing to the extended, shallow, featureless and solvent-accessible nature of the PP-binding interface [100]. Despite these limitations, PPI inhibition is receiving more and more attention in current pharmaceutical research. This is primarily because of the need to afford novel therapies in ever-complex diseases that are poorly affected by molecular regulation of standard protein classes. In the context of PPI modulation, their stabilization represents a possible alternative to impact pathophysiological conditions. The examples of PPI stabilization reviewed here would definitely support the technical feasibility of such an approach. From a medicinal chemistry perspective, it is noteworthy that a wide range of chemical structures can act as PPI stabilizers. These range from natural products (e.g. rapamycin) to typical drug-like and lead-like small molecules (e.g. tafamidis) and fragments (e.g. 1EBIO), suggesting possible opportunities for molecular intervention. These examples notwithstanding, our understanding of PPI stabilization and translatability to differentiated medicines is very much in its infancy, especially compared with established target class modulation and even PPI inhibition, where large knowledge gaps still exist. Similarly to more-established mode-of-actions, we could define a number of areas that require thorough investigation as research teams embark on PPI stabilization efforts. These can be conceptually centered on: (i) the targeted PPI(s); (ii) the link between the PPI(s) and the sought effect(s); and (iii) the link between the PPI(s) and toxicities. In the context of the targeted PPIs, screening feasibility, the relevance of the selected in vitro system and the stabilization interaction itself need to be considered. Accurately measuring stabilization between two partners is far more laborious than monitoring inhibition, and it often relies on biophysical methods and indirect measurements of association. Here, assay development activities need to focus on ensuring rapid turnaround of accurate data to enable chemical optimization of the PPI 
stabilizers. Because of the technical difficulties associated with stabilization assays, the protein partners are very rarely studied as a whole but are generally simplified using for example molecular biology techniques. Any such manipulation should not introduce in vitro artifacts or limit the ability to translate biophysical and biochemical results beyond the closed system employed. Lastly, a deeper understanding of the physical forces governing specific PPI stabilization could be advantageous. As researchers move away from simplified tactics like steric hindrance and functional isosterism, better appreciation of protein conformational aspects and resulting binding pocket plasticity is required. The cooperativity, additivity and/or synergy of protein oligomerization, its induction, development and time course are also areas worth exploring, especially with respect to kinetic aspects of ligand-induced stabilization.

The link between stabilization of a given PPI and the intended physiological effect requires continuous evaluation across systems of increased complexity, from recombinant protein models in vitro to whole animals in vivo. Special emphasis should be placed on relating in vitro measures of protein stabilization to the observed in vivo effect. This can include for example relative importance and regression analyses to identify the PPI stabilization parameters most descriptive of the effect in vivo. Incorporation of such parameters in pharmacokinetic-pharmacodynamic (PKPD) analyses would then be crucial to assess the true significance of PPI stabilization in vivo. Here, given the transient nature of protein-protein interfaces and their functional inference as well as the complex dynamics of associative and stabilization processes, adequate characterization of hysteresis would be advisable.

Similarly to exerting positive pharmacological effects, stabilization of PPIs could also lead to inappropriate toxicological response. These could be directly ascribed to the targeted PPI or could originate from concomitant PPI stabilization events. In the first case, investigation of potential effect discriminants (e.g. tissue and/or organ distribution, protein under- or over-expression, baseline state versus disease state) and accurate quantification of effect onset, intensity and duration alongside the therapeutic index are required. As discussed before, translating back this information to the PPI stabilization parameters and modality would be extremely informative to the optimization of PPI stabilizers. Because PPI networks show a certain degree of redundancy [101] and promiscuous proteins are required for network function and stability [102], it is conceivable to assume that a given PPI stabilizer could stabilize different PPIs. This raises the issue of PPI selectivity and/or promiscuity in the context of a biological and toxicological response. Efforts to investigate the potential for multiple PPI stabilization (e.g. bioinformatics and 3D structural information analyses, pathway analyses, system biology and chemogenomics approaches) and their experimental validation following the concepts highlighted before would support a more focused approach to the development of PPI stabilizers.

Finally, as in the case of other established mode-of-actions, nature has already demonstrated the potential for PPI stabilization: a feasible strategy to modulate protein function successfully that results in significant physiological responses. In addition to natural products, artificial and synthetic molecules have been found to modulate their target proteins by stabilizing inactive oligomeric states. Together with the still relatively small number of success stories of 'intended' PPI stabilization as mode-of-action, these examples make an important case for targeted PPI stabilization in drug discovery.

\section{References}

1 Azzarito, V. et al. (2013) Inhibition of $\alpha$-helix-mediated protein-protein interactions using designed molecules. Nat. Chem. 5, 161-173

2 Ivanov, A.A. et al. (2013) Targeting protein-protein interactions as an anticancer strategy. Trends Pharmacol. Sci. 34, 393-400

3 Wells, J.A. and McClendon, C.L. (2007) Reaching for high-hanging fruit in drug discovery at protein-protein interfaces. Nature 450, 1001-1009

4 Secchiero, P. et al. (2011) Recent advances in the therapeutic perspectives of nutlin-3. Curr. Pharm. Des. 17, 569-577

5 Vassilev, L.T. et al. (2004) In vivo activation of the p53 pathway by small-molecule antagonists of MDM2. Science 303, 844-848

6 Oltersdorf, T. et al. (2005) An inhibitor of Bcl-2 family proteins induces regression of solid tumours. Nature 435, 677-681

7 Rudin, C.M. et al. (2012) Phase II study of single-agent navitoclax (ABT-263) and biomarker correlates in patients with relapsed small cell lung cancer. Clin. Cancer Res.: Off. J. Am. Assoc. Cancer Res. 18, 3163-3169

8 Tse, C. et al. (2008) ABT-263: a potent and orally bioavailable Bcl-2 family inhibitor. Cancer Res. 68, 3421-3428

9 Christ, F. et al. (2010) Rational design of small-molecule inhibitors of the LEDGF/ p75-integrase interaction and HIV replication. Nat. Chem. Biol. 6, 442-448

10 Zimmermann, G. et al. (2013) Small molecule inhibition of the KRAS-PDEס interaction impairs oncogenic KRAS signalling. Nature 497, 638-642

11 He, M.M. et al. (2005) Small-molecule inhibition of TNF- $\alpha$. Science 310, 1022-1025

12 Filippakopoulos, P. et al. (2010) Selective inhibition of BET bromodomains. Nature 468, 1067-1073

13 Von Kleist, L. et al. (2011) Role of the clathrin terminal domain in regulating coated pit dynamics revealed by small molecule inhibition. Cell 146, $471-484$

14 Thiel, P. et al. (2012) Small-molecule stabilization of protein-protein interactions: an underestimated concept in drug discovery? Angew. Chem. Int. Ed 51, 2012-2018

15 Borel, J.F. et al. (1976) Biological effects of cyclosporin A: a new antilymphocytic agent. Agents Actions 6, 468-475

16 Zocher, R. et al. (1986) Biosynthesis of cyclosporin A: partial purification and properties of a multifunctional enzyme from Tolypocladium inflatum. Biochemistry $25,550-553$

17 Fricker, G. et al. (1996) Relevance of p-glycoprotein for the enteral absorption of cyclosporin A: in vitro-in vivo correlation. Br. J. Pharmacol. 118, 1841-1847

18 Wenger, R.M. et al. (1986) Cyclosporine: chemistry, structure-activity relationships and mode of action. In Metabolic Control in Diabetes Mellitus Beta Adrenoceptor Blocking Drugs NMR Analysis of Cancer Cells Immunoassay in the Clinical Laboratory Cyclosporine. pp. 157-191, Springer, Berlin/Heidelberg

19 Jeffery, J.R. (1991) Cyclosporine analogues. Clin. Biochem. 24, 15-21

20 Lazarova, T. and Weng, Z. (2003) Cyclosporin A analogues: recent advances. Expert Opin. Ther. Pat. 13, 1327-1332

21 Stähelin, H.F. (1996) The history of cyclosporin A (Sandimmune) revisited: another point of view. Experientia 52, 5-13

22 Handschumacher, R.E. et al. (1984) Cyclophilin: a specific cytosolic binding protein for cyclosporin A. Science 226, 544-547

23 Gallay, P.A. and Lin, K. (2013) Profile of alisporivir and its potential in the treatment of hepatitis C. Drug Des. Dev. Ther. 7, 105-115

24 Liu, J. et al. (1991) Calcineurin is a common target of cyclophilin-cyclosporin A and FKBP-FK506 complexes. Cell 66, 807-815

25 Huai, Q. et al. (2002) Crystal structure of calcineurin-cyclophilin-cyclosporin shows common but distinct recognition of immunophilin-drug complexes. Proc. Natl. Acad. Sci. U. S. A. 99, 12037-12042

26 Kino, T. et al. (1987) FK-506, a novel immunosuppressant isolated from a Streptomyces. I. Fermentation, isolation, and physico-chemical and biological characteristics. J. Antibiot. 40, 1249-1255

27 Goulet, M.T. et al. (1994) The medicinal chemistry of FK-506. Perspect. Drug Discov. Des. 2, 145-162 
28 Siekierka, J.J. et al. (1989) A cytosolic binding protein for the immunosuppressant FK506 has peptidyl-prolyl isomerase activity but is distinct from cyclophilin. Nature 341, 755-757

29 Griffith, J.P. et al. (1995) X-ray structure of calcineurin inhibited by the immunophilin-immunosuppressant FKBP12-FK506 complex. Cell 82, 507-522

30 Sehgal, S.N. et al. (1975) Rapamycin (AY-22,989), a new antifungal antibiotic. II. Fermentation, isolation and characterization. J. Antibiot. 28, 727-732

31 Martel, R.R. et al. (1977) Inhibition of the immune response by rapamycin, a new antifungal antibiotic. Can. J. Physiol. Pharmacol. 55, 48-51

32 Brown, E.J. et al. (1994) A mammalian protein targeted by G1-arresting rapamycin-receptor complex. Nature 369, 756-758

33 Choi, J. et al. (1996) Structure of the FKBP12-rapamycin complex interacting with the binding domain of human FRAP. Science 273, 239-242

34 Luengo, J.I. et al. (1995) Structure-activity studies of rapamycin analogs: evidence that the C-7 methoxy group is part of the effector domain and positioned at the FKBP12-FRAP interface. Chem. Biol. 2, 471-481

35 Bayle, J.H. et al. (2006) Rapamycin analogs with differential binding specificity permit orthogonal control of protein activity. Chem. Biol. 13, 99-107

36 Benjamin, D. et al. (2011) Rapamycin passes the torch: a new generation of mTOR inhibitors. Nat. Rev. Drug Discov. 10, 868-880

37 Giordanetto, F. and Kihlberg, J. (2014) Macrocyclic drugs and clinical candidates: what can medicinal chemists learn from their properties? J. Med. Chem. 57, 278-295

38 Bhat, S.V. et al. (1977) Structures and stereochemistry of new labdane diterpiniods from coleus forskohlii briq. Tetrahedron Lett. 18, 1669-1672

39 Seamon, K.B. et al. (1981) Forskolin: unique diterpene activator of adenylate cyclase in membranes and in intact cells. Proc. Natl. Acad. Sci. U. S. A. 78, 3363-3367

40 Sunahara, R.K. et al. (1997) Interaction of Gsalpha with the cytosolic domains of mammalian adenylyl cyclase. J. Biol. Chem. 272, 22265-22271

41 Zhang, G. et al. (1997) Structure of the adenylyl cyclase catalytic core. Nature 386, $247-253$

42 Tesmer, J.J. et al. (1997) Crystal structure of the catalytic domains of adenylyl cyclase in a complex with Gsalpha.GTPgammaS. Science 278, 1907-1916

43 Laurenza, A. et al. (1987) Stimulation of adenylate cyclase by water-soluble analogues of forskolin. Mol. Pharmacol. 32, 133-139

44 Tatee, T. et al. (1996) Forskolin derivatives. I. Synthesis, and cardiovascular and adenylate cyclase-stimulating activities of water-soluble forskolins. Chem. Pharm. Bull. 44, 2274-2279

45 Toya, Y. et al. (1998) Forskolin derivatives with increased selectivity for cardiac adenylyl cyclase. J. Mol. Cell. Cardiol. 30, 97-108

46 Pinto, C. et al. (2008) Activation and inhibition of adenylyl cyclase isoforms by forskolin analogs. J. Pharmacol. Exp. Ther. 325, 27-36

47 Ballio, A. et al. (1964) Fusicoccin: a new wilting toxin produced by Fusicoccum amygdali Del. Nature 203, 297

48 Sassa, T. et al. (1970) Isolation of a new plant growth substance with cytokinin-like activity. Nature 227, 379

49 Camoni, L. et al. (2013) The phytotoxin fusicoccin, a selective stabilizer of 14-3-3 interactions? IUBMB Life 65, 513-517

50 De Boer, A.H. and de Vries-van Leeuwen, I.J. (2012) Fusicoccanes: diterpenes with surprising biological functions. Trends Plant Sci. 17, 360-368

51 Milroy, L-G. et al. (2013) Stabilization and inhibition of protein-protein interactions: the 14-3-3 case study. ACS Chem. Biol. 8, 27-35

52 De Vries-van Leeuwen, I.J. et al. (2010) Fusicoccin-A selectively induces apoptosis in tumor cells after interferon-alpha priming. Cancer Lett. 293, 198-206

53 Camoni, L. et al. (2011) The phytotoxin fusicoccin promotes platelet aggregation via 14-3-3-glycoprotein Ib-IX-V interaction. Biochem. J. 436, 429-436

54 De Vries-van Leeuwen, I.J. et al. (2013) Interaction of 14-3-3 proteins with the estrogen receptor alpha F domain provides a drug target interface. Proc. Natl. Acad. Sci. U. S. A. 110, 8894-8899

55 Honma, Y. (2002) Cotylenin A - a plant growth regulator as a differentiationinducing agent against myeloid leukemia. Leuk. Lymphoma 43, 1169-1178

56 Kasukabe, T. et al. (2005) Effects of combined treatment with rapamycin and cotylenin A, a novel differentiation-inducing agent, on human breast carcinoma MCF-7 cells and xenografts. Breast Cancer Res. 7, R1097-R1110

57 Ottmann, C. et al. (2009) A structural rationale for selective stabilization of antitumor interactions of 14-3-3 proteins by cotylenin A. J. Mol. Biol. 386, 913-919

58 Molzan, M. et al. (2013) Stabilization of physical RAF/14-3-3 interaction by cotylenin A as treatment strategy for RAS mutant cancers. ACS Chem. Biol. 8, 1869-1875

59 Anders, C. et al. (2013) A semisynthetic fusicoccane stabilizes a protein-protein interaction and enhances the expression of $\mathrm{K}+$ channels at the cell surface. Chem. Biol. 20, 583-593
60 Kawakami, K. et al. (2012) A novel fusicoccin derivative preferentially targets hypoxic tumor cells and inhibits tumor growth in xenografts. Anticancer Agents Med. Chem. 12, 791-800

61 Toyomasu, T. et al. (2007) Fusicoccins are biosynthesized by an unusual chimera diterpene synthase in fungi. Proc. Natl. Acad. Sci. U. S. A. 104, 3084-3088

62 Arens, J. et al. (2013) Exploration of biosynthetic access to the shared precursor of the fusicoccane diterpenoid family. Chem. Commun. 49, 4337-4339

63 Johnson, C. et al. (2011) Visualization and biochemical analyses of the emerging mammalian 14-3-3-phosphoproteome. Mol. Cell. Proteomics MCP 10 M110.005751

64 Nalaskowski, M.M. et al. (2002) The human homologue of yeast ArgRIII protein is an inositol phosphate multikinase with predominantly nuclear localization. Biochem. J. 366, 549-556

65 Eckmann, L. et al. (1997) D-Myo-inositol 1,4,5,6-tetrakisphosphate produced in human intestinal epithelial cells in response to Salmonella invasion inhibits phosphoinositide 3-kinase signaling pathways. Proc. Natl. Acad. Sci. U. S. A. 94, 14456-14460

66 Watson, P.J. et al. (2012) Structure of HDAC3 bound to co-repressor and inositol tetraphosphate. Nature 481, 335-340

67 Ishida, R. et al. (1995) DNA topoisomerase II is the molecular target of bisdioxopiperazine derivatives ICRF-159 and ICRF-193 in Saccharomyces cerevisiae. Cancer Res. 55, 2299-2303

68 Weiss, G. et al. (1999) Dexrazoxane (ICRF-187). Gen. Pharmacol. Vasc. Syst. 32, $155-158$

69 Roca, J. et al. (1994) Antitumor bisdioxopiperazines inhibit yeast DNA topoisomerase II by trapping the enzyme in the form of a closed protein clamp. Proc. Natl. Acad. Sci. U. S. A. 91, 1781-1785

70 Classen, S. et al. (2003) Structure of the topoisomerase II ATPase region and its mechanism of inhibition by the chemotherapeutic agent ICRF-187. Proc. Natl. Acad. Sci. U. S. A. 100, 10629-10634

71 Johnson, S.M. et al. (2005) Native state kinetic stabilization as a strategy to ameliorate protein misfolding diseases: a focus on the transthyretin amyloidoses. Acc. Chem. Res. 38, 911-921

72 Connelly, S. et al. (2010) Structure-based design of kinetic stabilizers that ameliorate the transthyretin amyloidoses. Curr. Opin. Struct. Biol. 20, 54-62

73 Razavi, H. et al. (2003) Benzoxazoles as transthyretin amyloid fibril inhibitors: synthesis, evaluation, and mechanism of action. Angew. Chem. Int. Ed. Engl. 42, 2758-2761

74 Bulawa, C.E. et al. (2012) Tafamidis, a potent and selective transthyretin kinetic stabilizer that inhibits the amyloid cascade. Proc. Natl. Acad. Sci. U. S. A. 109, 9629 9634

75 Garrett, S.C. et al. (2008) A biosensor of S100A4 metastasis factor activation: inhibitor screening and cellular activation dynamics. Biochemistry 47, 986-996

76 Massie, S.P. (1954) The chemistry of phenothiazine. Chem. Rev. 54, 797-833

77 Jaszczyszyn, A. et al. (2012) Chemical structure of phenothiazines and their biological activity. Pharmacol. Rep. 64, 16-23

78 López-Muñoz, F. et al. (2005) History of the discovery and clinical introduction of chlorpromazine. Ann. Clin. Psychiatry 17, 113-135

79 Malashkevich, V.N. et al. (2010) Phenothiazines inhibit S100A4 function by inducing protein oligomerization. Proc. Natl. Acad. Sci. U. S. A. 107, 8605-8610

80 Noda, T. et al. (2006) Architecture of ribonucleoprotein complexes in influenza A virus particles. Nature 439, 490-492

81 Kao, R.Y. et al. (2010) Identification of influenza A nucleoprotein as an antiviral target. Nat. Biotechnol. 28, 600-605

$82 \mathrm{Su}$, C.-Y. et al. (2010) High-throughput identification of compounds targeting influenza RNA-dependent RNA polymerase activity. Proc. Natl. Acad. Sci. U. S. A. 107, 19151-19156

83 Gerritz, S.W. et al. (2011) Inhibition of influenza virus replication via small molecules that induce the formation of higher-order nucleoprotein oligomers. Proc. Natl. Acad. Sci. U. S. A. 108, 15366-15371

84 Wade, M. et al. (2010) The p53 orchestra: Mdm2 and Mdmx set the tone. Trends Cell Biol. 20, 299-309

85 Brown, C.J. et al. (2009) Awakening guardian angels: drugging the p53 pathway. Nat. Rev. Cancer 9, 862-873

86 Wade, M. and Wahl, G.M. (2009) Targeting Mdm2 and Mdmx in cancer therapy: better living through medicinal chemistry? Mol. Cancer Res. 7, 1-11

87 Graves, B. et al. (2012) Activation of the p53 pathway by small-molecule-induced MDM2 and MDMX dimerization. Proc. Natl. Acad. Sci. U. S. A. 109, 11788-11793

88 Xia, X.M. et al. (1998) Mechanism of calcium gating in small-conductance calcium-activated potassium channels. Nature 395, 503-507

89 Weston, A.H. et al. (2010) Impairment of endothelial SK(Ca) channels and of downstream hyperpolarizing pathways in mesenteric arteries from spontaneously hypertensive rats. Br. J. Pharmacol. 160, 836-843 
90 Chandy, K.G. et al. (1998) Isolation of a novel potassium channel gene hSKCa3 containing a polymorphic CAG repeat: a candidate for schizophrenia and bipolar disorder? Mol. Psychiatry 3, 32-37

91 Zhang, M. et al. (2012) Identification of the functional binding pocket for compounds targeting small-conductance $\mathrm{Ca}^{2+}$-activated potassium channels. Nat. Commun. 3, 1021

92 Pedarzani, P. and Stocker, M. (2008) Molecular and cellular basis of small - and intermediate - conductance, calcium-activated potassium channel function in the brain. Cell. Mol. Life Sci. 65, 3196-3217

93 Strøbæk, D. et al. (2004) Activation of human IK and SK Ca ${ }^{2+}$-activated K+ channels by NS309 (6,7-dichloro-1H-indole-2,3-dione 3-oxime). Biochim. Biophys. ActaBiomembr. 1665, 1-5

94 Gronemeyer, H. et al. (2004) Principles for modulation of the nuclear receptor superfamily. Nat. Rev. Drug Discov. 3, 950-964

95 DeManno, D. et al. (2003) Asoprisnil (J867): a selective progesterone receptor modulator for gynecological therapy. Steroids 68, 1019-1032
96 Madauss, K.P. et al. (2007) A structural and in vitro characterization of asoprisnil: a selective progesterone receptor modulator. Mol. Endocrinol. 21, 1066-1081

97 Germain, P. et al. (2009) Differential action on coregulator interaction defines inverse retinoid agonists and neutral antagonists. Chem. Biol. 16, 479-489

98 Le Maire, A. et al. (2010) A unique secondary-structure switch controls constitutive gene repression by retinoic acid receptor. Nat. Struct. Mol. Biol. 17, 801-807

99 Rose, R. et al. (2010) Identification and structure of small-molecule stabilizers of 14-3-3 protein-protein interactions. Angew. Chem. Int. Ed. Engl. 49, 4129-4132

100 Zinzalla, G. and Thurston, D.E. (2009) Targeting protein-protein interactions for therapeutic intervention: a challenge for the future. Future Med. Chem. 1, 65-93

101 Stelzl, U. et al. (2005) A human protein-protein interaction network: a resource for annotating the proteome. Cell 122, 957-968

102 Patil, A. et al. (2010) Hub promiscuity in protein-protein interaction networks. Int J. Mol. Sci. 11, 1930-1943 